\title{
Mise en place d'une expérience avec le grand public : entre recherche, vulgarisation et pédagogie
}

LOHEAC Youenn, ALIA Hayyan, BAZART Cécile, BCHIR Mohamed Ali, BLONDEL Serge, BONESCU Mihaela, BORNIER Alexandrine, BROUARD Joëlle, CHAPPE Nathalie, COCHARD François, FLAGE Alexandre, GALEOTTI Fabio, HOLLANDTS Xavier, HOPFENSITZ Astrid, JACQUEMET Nicolas, LE LEC Fabrice, LEFEBVRE Marianne, LEPLAT Mélody, MANTILLA Cesar, MATEU Guillermo, PERON Guillaume, PETERLE Emmanuel, PETIT Emmanuel, RAIBER Eva, ROSAZ Julie, ROZAN Anne, TISSERAND Jean-Christian, VILLEVAL Marie-Claire, WILLINGER Marc, ZYLBERSZTEJN Adam, SUTAN Angela ${ }^{1,2}$

Nous présentons la mise en place d'une expérience lors d'un évènement grand public national, de manière simultanée dans 11 villes françaises, en septembre 2015. L'expérience a impliqué plus de 2700 participants et a duré quatre heures ininterrompues. L'objectif de cet article est à la fois de fournir une feuille de route pour une éventuelle réplication et de penser à la manière dont la discipline peut investir des terrains nouveaux (vulgarisation, pédagogie populaire, communication grand public).

\section{METHODOLOGICAL CONSIDERATIONS ON IMPLEMENTING A PARTICIPATIVE EXPERIMENT}

We present the implementation of an economic experiment conducted simultaneously in 11 French cities, with over 2700 participants, during four uninterrupted hours, during a popular-science event held in September 2015. Our goal is both to provide a roadmap for a possible replication and to discuss how the discipline can invest in new fields (science popularization, popular education, public communication).

Mots-clés : expérience; vulgarisation; pédagogie; grand public.

JEL: A11, C93

\section{INTRODUCTION}

Dans le cadre de la Nuit Européenne des Chercheurs 2015, nous avons conduit une expérience d'économie comportementale, de manière simultanée dans 11 villes françaises. Dans cet article, nous présentons de manière synthétique et volontairement factuelle la mise en place de cette expérience, réalisée sous des objectifs et contraintes multiples, et qui est située à la frontière entre recherche scientifique, vulgarisation, pédagogie et action évènementielle. L'expérience a impliqué plus de 2700 participants et a duré quatre heures ininterrompues.

\footnotetext{
${ }^{1}$ Auteurs correspondants : youenn.loheac@brest-bs.com ; angela.sutan@escdijon.eu

${ }^{2}$ Institutions participantes : Université d'Angers, Univ. Bourgogne Franche-Comté ESC Dijon-CEREN, Brest Business School, Université de Bordeaux, Kedge Business School, Université de Montpellier, Toulouse School of Economics and Institute for Advanced Study in Toulouse, Université de Lyon, Paris School of Economics, ENGEES Strasbourg.

Les auteurs remercient la coordination nationale et les coordonnateurs locaux de la Nuit des Chercheurs, particulièrement Lionel Maillot et Meriem Fresson, et sont reconnaissants du soutien financier à travers l'appel "Grande Expérience Participative".
} 
En économie comportementale, les méthodes de collecte des données se sont diversifiées pour répondre à des besoins nouveaux engendrés par la production de nouvelles théories ou pour tester ou renforcer la validité externe des expériences de laboratoire (expériences de terrain, essais randomisés à grande échelle, expériences en contexte "naturel", expériences de neuroéconomie, etc.). En parallèle, la discipline a aussi investi le terrain de la pédagogie (Eber et Willinger, 2012), et les expériences réalisées en classe sont nombreuses dans la transmission des concepts économiques. Dans cet article, nous présentons une extension du domaine des possibles dans deux dimensions. La première dimension est celle du terrain de collecte de données, en profitant d'un événement grand public, permettant un recueil massif sur une courte période. La seconde dimension est celle de la pédagogie. L'économie expérimentale est ici utilisée afin de communiquer sur les mécanismes économiques non pas, comme c'est devenu classique, à destination des étudiants, mais à destination du grand public, et ce, afin d'échanger au sujet d'un thème contemporain. Cela permet de répondre aux faiblesses de diffusion et de sensibilisation des progrès de la science économique soulignées par le rapport Guesnerie (2008) sur l'enseignement des sciences économiques et sociales dans le secondaire.

Le projet a été mis en place en réponse à l'appel à projets lancé par les organisateurs de la Nuit Européenne des Chercheurs pour l'organisation d'une "Grande Expérience Participative" à conduire dans 11 villes françaises prédéfinies. Cette "Grande Expérience Participative" devait être un événement de rencontre ludique entre les chercheurs et le public. Le projet devait donc s'inscrire dans un cahier des charges décrivant quatre objectifs et définissant pour nous, les chercheurs, autant de contraintes : (i) en analysant la façon dont les individus allaient adapter leurs stratégies empiriques en présence de variations de traitement dans le protocole, notre travail devait remplir un objectif scientifique et académique, à savoir apporter des informations sur la prise de décisions ; (ii) cette expérience devait conduire à la diffusion de ces nouvelles connaissances à travers des publications spécialisées académiques et professionnelles en économie ; (iii) le projet devait constituer un transfert original de connaissances de la discipline vers un public non spécialisé, et constituer une occasion pour le public d'apprendre et d'être sensibilisé à des enjeux économiques actuels ; (iv) enfin, ce projet avait pour objectif une contribution méthodologique, c'est-à-dire la mise en œuvre de méthodes expérimentales valides sur un terrain "grand public", afin de partager ces méthodes et faciliter l'investigation de nouveaux terrains.

La plupart des articles de méthodologie expérimentale en économie identifient trois catégories d'objectifs poursuivis par l'économie expérimentale (selon Roth $(1986 ; 1988 ; 1995)$ : tester la théorie, rechercher des faits ou parler aux décideurs). Nous ajoutons, étant donné notre terrain, un objectif supplémentaire, celui de parler au "public", i.e. aux sujets expérimentaux, tout en respectant les canons de la discipline (contrôle expérimental, anonymat, incitations monétaires, etc.).

Mettre en place une expérience dans un contexte qui est à la fois terrain et scène implique la transmission d'une connaissance tacite au sens de Polanyi (1967), et donc, en parallèle avec la mise en place d'un protocole d'expérience, l'explication de ce protocole, des pratiques et compétences qui le composent et qui sont habituellement cachées à ceux qui ne sont pas experts. Il s'agissait ainsi de créer un cadre d'apprentissage expérientiel pour le public. Si l'on se réfère à la théorie de l'apprentissage de Kolb (1984), l'apprentissage expérientiel est efficace pour assurer cette transmission en cela que le spectateur devient aussi acteur de la prise de décision. Nous avons donc proposé une "approche expérientielle" au public, qui était en même temps notre sujet expérimental, et la rencontre a été basée sur trois principes : (i) l'implication du public dans l'échange avec le chercheur (par la participation à un jeu expérimental), (ii) une co-construction 
de la connaissance véhiculée dans les échanges (par l'explication à postériori de la construction, de la méthode et des objectifs de l'expérience), et (iii) un engagement fort du public dans cet apprentissage qu'il venait chercher dans un évènement de vulgarisation scientifique (à travers la mise en place d'incitations réelles à la prise de décision, comme dans une véritable séance de laboratoire, et le choix d'un sujet d'expérience auquel le public était susceptible d'être sensible).

Nous devions par conséquent déterminer pour cette expérience un thème qui puisse concilier ces divers objectifs et répondre aux nombreuses contraintes. Si une démarche d'économie expérimentale peut en soi contribuer à la transmission d'un savoir, nous avons trouvé important de confronter les sujets à un thème pour lequel les besoins pédagogiques existent. Ainsi, c'est le thème de la tragédie des biens communs qui a été choisi pour cette expérience, afin de traiter d'un sujet d'intérêt général permettant un échange aisé avec les participants. Notre expérience a donc été construite de manière à répondre aux contraintes de la discipline et aux contraintes d'un exercice particulier. Le protocole expérimental utilisé lors de la "Grande Expérience Participative» visait ainsi à reproduire, dans un contexte simplifié, la logique des décisions environnementales.

Cette expérience, ainsi que les méthodes mises en œuvre sont décrites dans cet article de la façon suivante. La première section traite du contexte et des contraintes expérimentales propres au protocole. La deuxième explique le choix de la tragédie des communs. La troisième section décrit la façon dont nous adaptons ce thème au terrain d'application. Le protocole expérimental et l'échantillon sont présentés dans la quatrième section. Enfin, nous concluons en tirant les enseignements de cette expérience de médiation scientifique recourant à l'économie expérimentale, et en présentant des éléments de partage avec le public après l'expérience.

\section{CONTEXTE ET CONTRAINTES EXPERIMENTALES}

Cette expérience a été retenue lors d'un appel à projet dans le cadre de la dixième Nuit Européenne des Chercheurs ${ }^{1}$, pour la France, intitulé "Grande Expérience Participative". Cet événement est une occasion unique de médiation scientifique, moins institutionnelle que la Fête de la Science, qui se déroule le soir, généralement dans un lieu emblématique. Elle permet au grand public de venir à la rencontre des chercheurs de leur ville et de les questionner sur leurs sujets, leurs pratiques, leur pensée, etc. Si dans les autres pays Européens, cette soirée a lieu uniquement dans une ville qui change tous les ans, en France elle a lieu simultanément dans onze villes : Angers, Besançon, Bordeaux, Brest, Dijon, Le Mans, Limoges, Lyon, Metz, Paris et Toulouse.

Le cahier des charges de la "Grande Expérience Participative" indique que cette expérience doit pouvoir être conduite dans toutes les villes concernées, auprès d'un grand nombre de personnes en une seule soirée. Elle doit également être compréhensible par tous et donner lieu à des restitutions, à la suite des sessions expérimentales, d'une part, et après l'expérience, dans le cadre de communications scientifiques, expertes et de vulgarisation, d'autre part.

Les contraintes logistiques étaient les suivantes. Premièrement, il s'agissait de rassembler une équipe de scientifiques capables de conduire l'expérience dans onze villes. Le public souhaite, en effet, rencontrer de véritables chercheurs, qui parlent de leurs recherches. Il fallait donc que

\footnotetext{
${ }^{1}$ Site internet de la Nuit Européenne des Chercheurs : http://www.nuitdeschercheurs-france.eu
} 
chacun d'entre nous maitrise le domaine de l'expérience. Deuxièmement, il fallait mettre en place un protocole expérimental court, compréhensible par tous rapidement (la Nuit des Chercheurs est un évènement très grand public, qui accueille des visiteurs de tous âges), et qui ne demandait pas la mise en place de procédures informatiques compliquées (dans certaines villes, nous ne pouvions pas assurer une expérience à travers un programme informatique, du fait de l'affluence attendue et de la disponibilité des équipements, et pour ne pas limiter l'accès aux seuls détenteurs de smartphones, par exemple). Troisièmement, il était nécessaire que l'expérience permette un échange au sujet de son objet et de sa finalité. Les participants venaient surtout pour apprendre et pas uniquement pour jouer le rôle de cobayes. Quatrièmement, il nous fallait respecter les canons de la méthode expérimentale, afin que les productions issues de cette expérience puissent donner lieu à publication dans des revues scientifiques de référence. Enfin, il fallait mettre en place un protocole suffisamment flexible pour s'adapter à un nombre incertain et variable de participants par session. En effet, l'expérience allait avoir lieu dans des endroits très différents (grands amphithéâtres pouvant accueillir des centaines de personnes, petites salles avec un nombre fixe de personnes par session, places publiques, etc.). Nous devions donc prévoir une expérience qui pouvait être réalisée avec des sessions de tailles différentes. Le public de la Nuit des Chercheurs visite les ateliers des chercheurs au gré de leurs envies. Il se pouvait ainsi qu'une session se déroulât avec quatre visiteurs seulement et une autre avec 400 , en fonction de l'affluence.

Ainsi, la première étape a consisté à rassembler des chercheurs pouvant contribuer à la conception du protocole et assurer la mise en œuvre de l'expérience dans les différentes villes. La mobilisation a été réalisée à travers un appel au sein des chercheurs membres de l'ASsociation Française d'Economie Expérimentale (ASFEE). Les principes (et contraintes méthodologiques et matérielles) de l'expérience ont été ensuite présentés aux coordinateurs de la Nuit Européenne des Chercheurs de chaque ville.

Une partie de l'équipe rassemblée s'est ensuite chargée de préparer le protocole expérimental. Partant d'une idée de gestion de ressources naturelles communes, notre objectif était d'aboutir à un protocole simple et compréhensible par tous et permettant de produire une connaissance scientifique originale. Cette partie a donné lieu à un très grand nombre d'échanges et de discussions sur le type de jeu testé et sur les différents paramètres du protocole expérimental : les différents traitements, la définition de l'ensemble des décisions possibles pour les participants, le niveau d'information diffusé dans les différents traitements, le niveau de contextualisation et de réalisme, les incitations mises en œuvre. L'introduction d'une incitation réelle a fait l'objet de longues discussions. Nous avons opté pour des bons d'achats de 10 euros dont le rôle sera expliqué après la justification du choix du thème scientifique.

Nous avons ainsi abouti à un protocole expérimental dont les instructions tenaient sur une seule feuille et qui allaient être détaillées à l'oral par les expérimentateurs à l'aide d'une présentation power point. L'expérience a été conçue pour durer de 15 à 20 minutes et laisser une dizaine de minutes pour un échange avec les participants, sachant que la durée totale maximale d'une session fixée par les organisateurs de la Nuit des Chercheurs était de 30 minutes.

\section{LE CHOIX DU THEME EXPERIMENTAL : LA TRAGEDIE DES BIENS COMMUNS}

La tragédie des biens communs est un des jeux "classiques" de l'économie expérimentale. L'exemple le plus standard de la tragédie des communs est l'épuisement des ressources naturelles communes du fait de leur surexploitation. Qu'il s'agisse de ressources halieutiques ou forestières, 
de minerais ou d'eau, une exploitation plus intense que la vitesse de reproduction de la ressource conduit au fil du temps à sa disparition. Nous avons choisi ce thème parce qu'il était familier à l'ensemble des chercheurs. De plus, de nombreuses expériences ont été conduites avec succès à ce sujet dans de nombreux contextes (du laboratoire à des terrains avec les personnes directement concernées, voir par exemple Cardenas et al. 2013 ; Ostrom, 2006). Nous pouvions donc espérer que notre tâche allait être facilitée par une réception favorable du public. Nous avons aussi choisi ce thème parce qu'il constitue toujours un sujet d'actualité qui concerne les citoyens. Un élément déterminant de notre choix a aussi été le fait que, depuis Gordon (1954), le problème de la tragédie des biens communs est formalisé. Il résulte en effet de la volonté de chacun de maximiser son gain individuel au détriment de la recherche d'une solution socialement optimale. C'était donc un cadre idéal que nous pouvions exposer au public afin de lui montrer que les comportements font l'objet de modélisations et peuvent être anticipés de manière théorique en économie comportementale. De plus, ce problème est un sujet pris en charge par les décideurs publics ou privés (élus politiques, représentants de la société civile, etc.), qui cherchent à agir sur les variables susceptibles d'accroître la propension des individus à se diriger vers une autogouvernance durable (Ostrom, 1999).

Afin de conserver un protocole simple et qui parle au public de la Nuit Européenne des Chercheurs, nous nous sommes focalisés sur trois variables susceptibles d'influences les décisions dans notre expérience. La première variable est le taux de renouvellement des ressources (faible ou fort - cet élément est facilement intégrable par le grand public qui connait dans la nature des ressources épuisables qui se renouvellent rapidement ou lentement). La deuxième variable est l'information dont disposent les individus sur les seuils de soutenabilité lors de l'exploitation de ces ressources (le public est sensibilisé par les décideurs et les médias sur les points de non-retour dans les l'exploitation des forêts, par exemple). Enfin, la troisième variable est l'échelle de disponibilité de la ressource, i.e. son caractère local ou global (l'expérience allait avoir lieu dans 11 villes françaises, et le public en avait connaissance, ainsi, la dimension locale et globale des décisions était un avantage à prendre en compte et aussi un élément facile à expliquer).

\section{LE PROTOCOLE EXPERIMENTAL : ADAPTATION DU THEME SCIENTIFIQUE AU TERRAIN D'APPLICATION}

Dans le cadre des contraintes présentées précédemment, nous avons été conduits à faire des choix dans notre protocole et à hiérarchiser l'importance des canons d'économie expérimentale à respecter.

Une première adaptation vis-à-vis des règles habituelles de réalisation des expériences a consisté à contextualiser la situation de prise de décision. Après avoir réalisé plusieurs pilotes du protocole, nous avons conclu que ce format serait plus facile à transmettre au public. Ainsi, les participants se voient attribuer un rôle et sont exposés à une ressource naturelle au niveau local et au niveau national. Ils sont ainsi soit des bucherons, soit des pêcheurs. S'ils sont des bucherons, ils peuvent couper des arbres à la fois dans un bois auquel seuls les 10 bucherons de leur ville peuvent accéder à chaque génération et dans une forêt à laquelle les 100 bucherons du pays ont accès à chaque génération. S'ils sont des pêcheurs, ils peuvent pêcher des poissons à la fois dans un lac auquel seuls les 10 pêcheurs de leur ville peuvent accéder à chaque génération et dans la mer à laquelle les 100 pêcheurs du pays ont accès à chaque génération.

Dans le cadre de l'expérience, les participants étaient répartis aléatoirement entre quatre traitements et répondaient à deux questions (voir Tableau 1). Les traitements sont le croisement des informations sur le taux de reproduction de la ressource naturelle et sur le taux de soutenabilité de l'extraction de cette ressource. Chaque participant choisit dans le contexte auquel 
il est assigné le nombre d'unités (entre 0 et 5) de la ressource naturelle qu'il souhaite extraire au niveau local et au niveau national. Les fiches de décision des participants sont présentées en annexe.

Il est précisé que, à l'issue de l'expérience, les participants seront placés aléatoirement dans des générations de 100 personnes au niveau national et de 10 personnes au niveau local. Ils ne savent pas à quelle génération ils appartiennent, ni combien de générations il y a avant eux et après eux. La seule information est qu'il peut y avoir une génération avant eux et une génération après eux. Ceci reproduit des situations réelles d'épuisement de ressources dans lesquelles les citoyens ont généralement connaissance du caractère épuisable de la ressource, mais ne savent pas exactement quel est le stock disponible, ni depuis combien de temps la ressource est exploitée, ni s'ils sont les premiers à l'exploiter (situation d'abondance) ou, au contraire, s'ils sont parmi les derniers à en bénéficier. Cette astuce du protocole nous permet aussi de faire face à la contrainte liée à la taille des sessions. Habituellement, en matière de jeux sur les prélèvements des ressources naturelles, les sessions sont conduites avec un nombre fixe d'individus. Notre variation nous permet de reconstituer des générations à postériori, et donc de pouvoir conduire des sessions avec des nombres de participants différents à la fois entre les villes et d'une session à l'autre au sein d'une même ville.

Ce qui distingue les deux ressources naturelles est leur niveau de reproduction entre deux générations : $5 \%$ pour les arbres et $15 \%$ pour les poissons. Ceci mime la réalité (un arbre arrive à maturité plus difficilement qu'un poisson) et est facilement compréhensible par le public. Par ailleurs, quelle que soit la ressource, le stock initial est de 230 unités au niveau local (lac et bois) et de 2300 unités au niveau national (mer et forêt). Cela nous permet de comparer les prélèvements dans chaque traitement et contexte de façon symétrique.

Un traitement informationnel est ajouté. En effet, dans certaines sessions, nous informons les participants sur le taux de soutenabilité des ressources naturelles, c'est-à-dire le niveau d'extraction permettant à la génération suivante de bénéficier du même stock de ressource que la génération actuelle. Que ce soit au niveau local ou au niveau national, ce taux est d'un arbre par bucheron par génération et de 3 poissons par pêcheur et par génération. Cet élément mime l'existence d'annonces publiques sur les points de non-retour.

Tableau 1. Synthèse des traitements

Sans information

Bucherons / Arbres

100 bûcherons aux abords de la forêt

Taux de reproduction : 5\%

Pêcheurs / Poisons

(PSI)

10 pêcheurs autour du lac 100 pêcheurs aux abords de la mer

Taux de reproduction : 15\%
Avec information

(AAI)

10 bûcherons autour du bois 100 bûcherons aux abords de la forêt Taux de reproduction : $5 \%$ Niveau de soutenabilité : 1 arbre

(PAI)

10 pêcheurs autour du lac 100 pêcheurs aux abords de la mer Taux de reproduction : 15\% Niveau de soutenabilité : 3 poissons

Chaque participant pouvait extraire entre 0 et 5 unités de ressource au niveau local et entre 0 et 5 unités de la même ressource au niveau national. Ils savaient qu'ils partageaient cette expérience avec d'autres participants présents dans d'autres villes de France au même moment (la liste des 
villes leur est communiquée). En termes d'incitation, chaque unité de ressource extraite donne le droit de participer à un tirage au sort lors duquel 300 bons d'achats de 10 euros sont à gagner.

Si l'incitation repose en partie sur du hasard, elle n'en est pas moins réelle et crédible (à en juger par les commentaires qui nous ont été fait par des participants). Dans ce cadre, nous indiquons que les participants sont ordonnés au sein de leur génération, que ce soit au niveau local ou au niveau national. Cela a une conséquence sur les unités de ressources éligibles au tirage au sort. En effet, s'il y a assez de poissons ou d'arbres pour sa génération, chaque participant reçoit autant de tickets de loterie qu'il a extrait de poissons ou d'arbres. En revanche, s'il n'y a pas assez de poissons et d'arbres pour toute sa génération, le rang du participant au sein de sa génération est pris en compte. Les premiers reçoivent autant de tickets de loterie qu'ils ont pris de poissons ou d'arbres, puis un participant reçoit moins de tickets de loteries que ce qu'il a pris en poissons ou en arbres, et les derniers ne reçoivent aucun ticket de loterie. Enfin, si la génération précédente n'a laissé aucun poisson ou arbre, alors les participants de la génération concernée ne reçoivent aucun ticket de loterie.

\section{DEROULEMENT DE L'EXPERIENCE ET PARTICIPANTS}

Chaque session expérimentale s'est déroulée de la façon présentée dans le Tableau 2, selon une procédure classique en laboratoire.

Tableau 2. Procédures

\begin{tabular}{|c|c|}
\hline Étape & Activité \\
\hline Étape 1 & $\begin{array}{l}\text { Accueil des participants avec distribution d'une fiche d'instructions et de réponse } \\
\text { et de crayons }\end{array}$ \\
\hline Étape 2 & Lecture des instructions présentant le contexte et les rôles des participants \\
\hline Étape 3 & Explication du principe de génération \\
\hline Étape 4 & Description des stocks disponibles au démarrage de l'expérience \\
\hline Étape 5 & Description des décisions à prendre \\
\hline Étape 6 & Explication de la reproduction des ressources entre deux générations \\
\hline Étape 7 & Information sur le taux de soutenabilité, le cas échéant \\
\hline Étape 8 & Description et explication des incitations \\
\hline Étape 9 & $\begin{array}{l}\text { Prise de décision par les participants, questionnaire post-expérimental et collecte } \\
\text { des fiches }\end{array}$ \\
\hline Étape 10 & Information des participants sur le mode de publication des résultats \\
\hline Étape 11 & $\begin{array}{l}\text { Échange avec les chercheurs présents au sujet de la méthode expérimentale, de } \\
\text { l'expérience, de la tragédie des communs, de l'économie (sans présenter les } \\
\text { résultats attendus du protocole) }\end{array}$ \\
\hline Étape 12 & Évacuation de la salle. \\
\hline
\end{tabular}

Les participants ont été recrutés parmi les gens qui se rendaient à la Nuit Européenne des Chercheurs selon différentes modalités. Dans certaines villes, il était annoncé que les visiteurs pouvaient participer librement à des sessions d'expérience toutes les demi-heures. Lorsque l'expérience se déroulait dans un grand amphithéâtre, nous acceptions dans la salle tous les visiteurs intéressés. Si la salle avait une capacité limitée, les visiteurs étaient invités à revenir plus tard dans la soirée. Dans d'autre villes, un nombre limité de sessions avec un nombre fixe de places était prévu dans la soirée, à des heures précises. Les visiteurs s'inscrivaient sur ces créneaux-là. 
2813 personnes ont participé à cette expérience le 25 septembre 2015. 2723 personnes ont répondu aux quatre questions essentielles pour l'analyse (extraction locale, extraction nationale, âge, sexe), soit 96,8\% des participants. La moyenne d'âge est de 28 ans (allant de 2 à 91 ans), avec des différences entre villes, et $55 \%$ des participants sont des femmes. Le Tableau 3 détaille le nombre de participants et d'observations par ville, ainsi que les âges moyens et la proportion de femmes. Globalement, la répartition entre les quatre traitements est équilibrée. Entre 597 et 784 personnes ont répondu dans chaque condition de contexte et d'information.

Tableau 3. Effectifs et description des participants par ville

\begin{tabular}{lcccccccc} 
& Part. & Obs. & Age & Femmes & $\begin{array}{c}\text { Obs. } \\
\text { PSI }\end{array}$ & $\begin{array}{c}\text { Obs. } \\
\text { PAI }\end{array}$ & $\begin{array}{c}\text { Obs. } \\
\text { ASI }\end{array}$ & $\begin{array}{c}\text { Obs. } \\
\text { AAI }\end{array}$ \\
\hline France & 2813 & 2723 & 28.0 & 0.552 & 746 & 623 & 764 & 590 \\
Angers & 207 & 202 & 31.8 & 0.519 & 50 & 51 & 53 & 48 \\
Besançon & 224 & 221 & 28.2 & 0.583 & 52 & 53 & 62 & 54 \\
Bordeaux & 303 & 273 & 23.3 & 0.582 & 77 & 59 & 84 & 53 \\
Brest & 726 & 698 & 26.4 & 0.584 & 177 & 182 & 182 & 157 \\
Dijon & 487 & 478 & 29.5 & 0.546 & 142 & 97 & 142 & 97 \\
Le Mans & 123 & 122 & 35.1 & 0.475 & 51 & 10 & 53 & 8 \\
Limoges & 122 & 122 & 24.9 & 0.377 & 31 & 29 & 32 & 30 \\
Lyon & 168 & 165 & 28.1 & 0.563 & 54 & 31 & 48 & 32 \\
Metz & 129 & 128 & 30.0 & 0.625 & 23 & 41 & 23 & 41 \\
Paris & 126 & 117 & 31.1 & 0.461 & 39 & 21 & 37 & 20 \\
Toulouse & 198 & 197 & 26.8 & 0.568 & 50 & 49 & 48 & 50 \\
\hline \multicolumn{7}{c}{ Note: Part. = Participants ; Obs. = Observations ; PSI = poissons sans information ; PAI = poissons avec } \\
information ; ASI = arbres sans infomation ; AAI = arbres avec information. &
\end{tabular}

L'analyse des données donnera lieu à une publication scientifique ultérieure, ainsi qu'à une diffusion sur le blog de la « Grande Expérience Participative» au sein du site internet de la Nuit Européenne des Chercheurs.

\section{CONCLUSION : UNE EXPERIENCE DE MEDIATION SCIENTIFIQUE}

La réalisation de cette expérience nous a permis, outre la partie académique classique, de nous plonger dans une expérience de vulgarisation scientifique. Ceci permet de faire la transition de la production de données expérimentale à la description de ce processus. Cette transition implique la prise en compte de la critique de Collins (1985) qui stipule qu'elle est possible uniquement lorsque, de manière explicite, la distinction peut être faite entre une "bonne" et une "mauvaise" expérience. Cette distinction doit être crédible et internalisée à la fois par les chercheurs de la discipline, dans la construction du protocole, mais aussi à l'extérieur de la discipline, par le public, qui doit comprendre nos choix. En économie expérimentale, plusieurs normes et pratiques unanimement acceptées par la communauté existent : la règle de non-tromperie des participants, l'existence des incitations, la connaissance commune des instructions, etc. Ceci facilite la tâche en termes de partage des procédures mises en œuvre, mais nous oblige à faire des choix. La "Grande Expérience Participative" a été un événement unique, et l'exercice s'est situé à la croisée d'ambitions multiples (construire un protocole valide, récolter des données scientifiquement utilisables, vulgariser la méthode, vulgariser le thème, responsabiliser le public, etc.) et de contraintes logistiques, méthodologiques et institutionnelles. Nous avons donc entrepris d'expliquer comment nous les avons concilié et avons fait des choix, en présentant, dans un 
premier temps, les principes de l'économie expérimentale et comportementale, et, dans un deuxième temps, comment nous avons construit le protocole pour pouvoir les respecter.

Au-delà de cet effort de vulgarisation, cette expérience nous a permis, d'une part, de nous interroger sur l'importance relative des principes de l'économie expérimentale : doit-on privilégier, si des choix sont à faire, la neutralité du contexte, les incitations monétaires, le contrôle, la non-tromperie des participants ? En effet, Guala (2005) précise que le savoir expérimental est construit, et qu'il est le fruit de règles et conventions qui doivent être reproduites, quel que soit le terrain de l'expérience. À ce titre, d'une part, Friedman and Sunder (1994) stipulent par exemple que l'économie expérimentale utilise des données créées dans des conditions contrôlées. Le contrôle constitue ainsi une des contraintes fortes à remplir lorsque le laboratoire est déplacé dans un événement de médiation scientifique. Avec un design astucieux, nous avons réussi à conserver tous ces principes dans notre protocole, en relâchant seulement la neutralité du contexte, et sans introduire un biais expérimental de demande (parce que la situation évoquée correspond à une situation très proche de la prise de décision réelle). D'autre part, ce protocole n'a pas été seulement porteur de contraintes, mais aussi d'opportunités. En effet, nous avons pu explorer un protocole générationnel qui n'était pas réalisable facilement en laboratoire, une situation d'incertitude sur la place de l'individu au sein d'une génération et de cette génération au fil du temps, ainsi que la distinction réelle par ville et par ressource locale-nationale. Enfin, nous avons pu collecter un nombre record de données (2700 participants) en quelques heures.

De ce fait, la question de la sélection de notre échantillon s'est posée. D'après les statistiques de fréquentation de la Nuit des Chercheurs, l'évènement est réellement "grand public": il n'y a pas de surreprésentation d'une tranche d'âge, d'un sexe, d'une catégorie socio-professionnelle. Enfin, notre échantillon est inégalement réparti en fonction des villes. Ceci est dû à une organisation très différente de l'événement en fonction de la ville : si à Brest l'endroit accueille plus de 4000 visiteurs dans la soirée, à Paris l'événement est volontairement conservé "petit" par les organisateurs.

Lors du débriefing avec le public, la mission pédagogique a consisté à introduire aussi le dilemme du bien commun, la tragédie des communs, en plus des éléments sur la méthodologie de l'économie expérimentale, et plus généralement sur les sciences économiques (en répondant à des questions telles que "qu'est-ce que l'économie ?"). D'autre part, cet objectif rejoint l'utilisation de l'économie expérimentale comme outil à visée pédagogique (Eber et Willinger, 2012). De façon générale, l'expérience est l'occasion de présenter le mode de penser de l'économiste à travers les concepts d'arbitrage, d'opportunité, d'optimisation, en faisant réfléchir les participants sur la façon dont ils ont agi (sans être normatif). L'approche par une expérience vécue permet également d'aborder les questions de reproductibilité des expériences, notamment à travers les contraintes de contrôle, ainsi que des comparaisons des différentes expériences portant sur le même sujet.

De plus, nous avons insisté sur le fait que, dans un contexte de raréfaction des ressources disponibles, l'examen des potentialités offertes par la mobilisation du "capital comportemental" des individus peut être un outil efficace pour améliorer la compréhension de la résistance des acteurs aux changements, notamment afin de favoriser la transition écologique. Nous avons introduit des exemples sur les leviers comportementaux susceptibles d'être activés afin de lever cette résistance et d'aboutir à des modifications comportementales durables. Pour ceci, nous avons repris la définition d'utilisation et de préservation de l'environnement de Milfont et Duckitt (2010), selon laquelle "la préservation de l'environnement exprime la croyance générale que la priorité doit être donnée à la protection de la nature et la diversité des espèces animales dans leur état naturel, et les préserver de l'utilisation et l'altération par l'être humain", alors que l'utilisation 
de l'environnement exprime "la croyance générale qu’il est normal, approprié et nécessaire pour la nature, et tous les phénomènes et espèces naturels d'être utilisés et altérés pour répondre aux objectifs humains." Ceci nous a permis de discuter d'éco-comportement avec le public, d'échanger au sujet des intérêts collectifs et personnels. Il s'agissait d'expliquer que, l'individu va devoir effectuer une exploitation "raisonnable" de l'environnement, qui lui permettra non seulement de subvenir à ses besoins, mais qui pourra aussi permettre à des individus actuels ou futurs susceptibles de partager les mêmes ressources, de subvenir à leurs propres besoins. La pérennité des ressources doit ainsi être assurée sur le long terme. En surexploitant l'environnement, l'individu "non seulement diminue les possibilités offertes aux autres individus d'obtenir une part raisonnable de ressources, mais il impacte également la capacité de l'environnement à se régénérer sur le long terme" (Adjukovic, 2015), et donc empiète sur la capacité des générations futures de prélever des ressources.

Nous avons enfin discuté avec les participants du dilemme de l'exploitation : alors que de nombreuses ressources sont communes, aucun utilisateur ne peut être privé de leur utilisation, même abusive (par exemple : la faune, les ressources en eau, l'air pur, etc.). Ceci conduit à la "tragédie des biens communs" lorsque le comportement humain est entraîné par la maximisation des gains individuels et non par le désir de parvenir à une solution socialement optimale. Alors que les enquêtes confirment un désir largement répandu dans la population de prendre soin de telles ressources, des preuves empiriques de plus en plus alarmantes montrent leur surexploitation, pour certaines, à un point de non-retour. En économie expérimentale, à travers des jeux incitatifs, nous avons expliqué pouvoir faire la différence entre ce que les gens déclarent et ce qu'ils font et ainsi éviter le biais déclaratif.

\section{REFERENCES BIBLIOGRAPHIQUES}

Adjukovic (2015). Appropriation et valorisation sociale de l'orientation pro-environnementale, thèse de doctorat, Université de Bourgogne.

Cardenas, J., Janssen, M., and Bousquet, F. (2013). Handbook on Experimental Economics and the Environment., chapter Dynamics of Rules and Resources: Three New Field Experiments on Water, Forests and Fisheries. Edward Elgar Publisher.

Collins, H.M. (1985). Changing Order, Beverly Hills, Sage.

Eber, N. Willinger, M. (2012). L'économie expérimentale, La Découverte.

Friedman, D., Sunder, S. (1994). Experimental Methods: A Primer for Economists, Cambridge, Cambridge University Press.

Gordon, J. S. (1954). The economic theory of a common-property resource: The fishery. Journal of Political Economy 62: 124-142.

Guala, F. (2005). The methodology of experimental economics. Cambridge University Press.

Guesnerie, R. (2008). Rapport au Ministre de l'Éducation nationale de la mission d'audit des manuels et programmes de sciences économiques et sociales du lycée.

Kolb, D. A. (1984). Experiential learning: Experience as the source of learning and development. New Jersey: Prentice-Hall.

Milfont, T. L., Duckitt, J. (2010). The environmental attitudes inventory: A valid and reliable measure to assess the structure of environmental attitudes. Journal of Environmental Psychology, 30(1), 80-94.

Ostrom, E. (1999). Coping with tragedies of the commons. Annual review of political science 2.1: 493-535.

Ostrom, E. (2006). The value-added of laboratory experiments for the study of institutions and common-pool resources. Journal of Economic Behavior and Organization, 61:149-163.

Polanyi, M. (1967). The Tacit Dimension, New York, Anchor. 
Roth, A.E. (1986). Laboratory Experimentation in Economics", Economics and Philosophy, 2, 245-273.

Roth, A.E. (1988). Laboratory Experimentation in Economics: A Methodological Overview, Economic Journal, 98, 974-1031.

Roth, A.E. (1995). "Introduction to Experimental Economics", in J.H. Kagel and A.E. Roth (eds.) The Handbook of Experimental Economics, Princeton, Princeton University Press.

\section{ANNEXE. Exemple d'instructions, Traitements sans information}

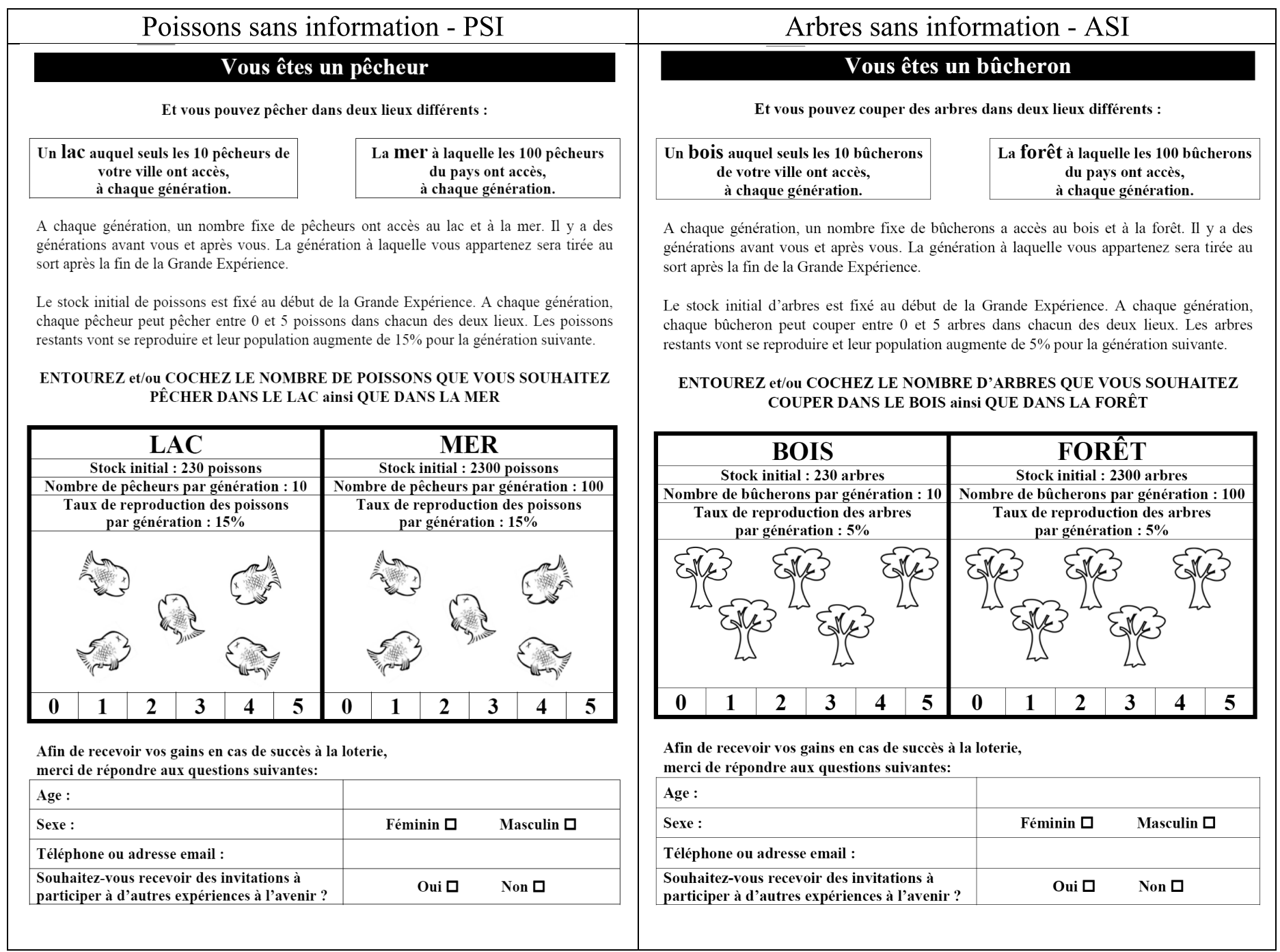

